\title{
An alternative route of bacterial infection associated with a novel resistance locus in the Daphnia-Pasteuria host-parasite system
}

\author{
Gilberto Bento $^{1} \cdot$ Peter D. Fields ${ }^{1} \cdot$ David Duneau $^{1,2} \cdot$ Dieter Ebert $^{1}{ }^{1}$
}

Received: 12 September 2019 / Revised: 7 June 2020 / Accepted: 7 June 2020 / Published online: 19 June 2020

(c) The Author(s) 2020. This article is published with open access

\begin{abstract}
To understand the mechanisms of antagonistic coevolution, it is crucial to identify the genetics of parasite resistance. In the Daphnia magna-Pasteuria ramosa host-parasite system, the most important step of the infection process is the one in which $P$. ramosa spores attach to the host's foregut. A matching-allele model (MAM) describes the host-parasite genetic interactions underlying attachment success. Here we describe a new P. ramosa genotype, P15, which, unlike previously studied genotypes, attaches to the host's hindgut, not to its foregut. Host resistance to P15 attachment shows great diversity across natural populations. In contrast to $P$. ramosa genotypes that use foregut attachment, P15 shows some quantitative variation in attachment success and does not always lead to successful infections, suggesting that hindgut attachment represents a less-efficient infection mechanism than foregut attachment. Using a Quantitative Trait Locus (QTL) approach, we detect two significant QTLs in the host genome: one that co-localizes with the previously described $D$. magna PR locus of resistance to foregut attachment, and a second, major QTL located in an unlinked genomic region. We find no evidence of epistasis. Fine mapping reveals a genomic region, the D locus, of $\sim 13 \mathrm{~kb}$. The discovery of a second $P$. ramosa attachment site and of a novel host-resistance locus increases the complexity of this system, with implications for both for the coevolutionary dynamics (e.g., Red Queen and the role of recombination), and for the evolution and epidemiology of the infection process.
\end{abstract}

\section{Introduction}

Host-parasite interactions are thought to be one of the main drivers of organismic evolution, promoting both diversification and genetic diversity (Schmid-Hempel 2011). The theory is that hosts evolve to minimize fitness costs associated with parasitism, whereas parasites evolve to maximize fitness while exploiting the host and avoiding its defense mechanisms. Different evolutionary models have

Associate editor: Gerald Heckel

Supplementary information The online version of this article (https:// doi.org/10.1038/s41437-020-0332-x) contains supplementary material, which is available to authorized users.

Dieter Ebert

dieter.ebert@unibas.ch

1 Department of Environmental Sciences, Zoology, University of Basel, Vesalgasse 1, 4051 Basel, Switzerland

2 Université Toulouse 3 Paul Sabatier, CNRS, UMR5174, EDB (Laboratoire Évolution \& Diversité Biologique), Toulouse, France been proposed to underlay such host-parasite coevolution, including negative frequency-dependent selection (NFDS), selective sweeps, and heterozygote advantage (Ebert 2008; Wilfert and Jiggins 2013; Papkou et al. 2016). However, as few naturally coevolving host-parasite systems have been sufficiently explored, the genetic mechanisms of these systems are largely unknown (Tiffin and Moeller 2006; van Oosterhout 2009; Ejsmond and Radwan 2015, Wilfert and Jiggins 2013, Bento et al. 2017).

Parasite infection is a complex process that typically requires multiple steps for successful completion of the parasite's life cycle, i.e., infection, within-host reproduction, and transmission (Hall et al. 2017). With each step, the parasite must overcome the host's defense mechanisms to continue its life cycle, whereas the host's degree of success at each step results in different fitness and evolutionary outcomes. A single infection-blocking step can render the host totally resistant, even if the other steps would allow the parasite to proceed. Identifying and studying specific infection steps can, thus, illuminate the complex attack and defense portfolios of specific host-parasite coevolutionary interactions (Hall et al. 2017, Lievens et al. 2018). 
Fig. 1 Attachment of Pasteuria ramosa to Daphnia magna host. a $D$. magna host with attachment sites for P. ramosa spores (green dots) attached to the foregut and hindgut, but not to the gut midsection (darkgreen lining). $P$. ramosa spores marked with a green fluorescent dye (and indicated by white arrows) may attach to the foregut (b) or to the hindgut (c) of the D. magna host.

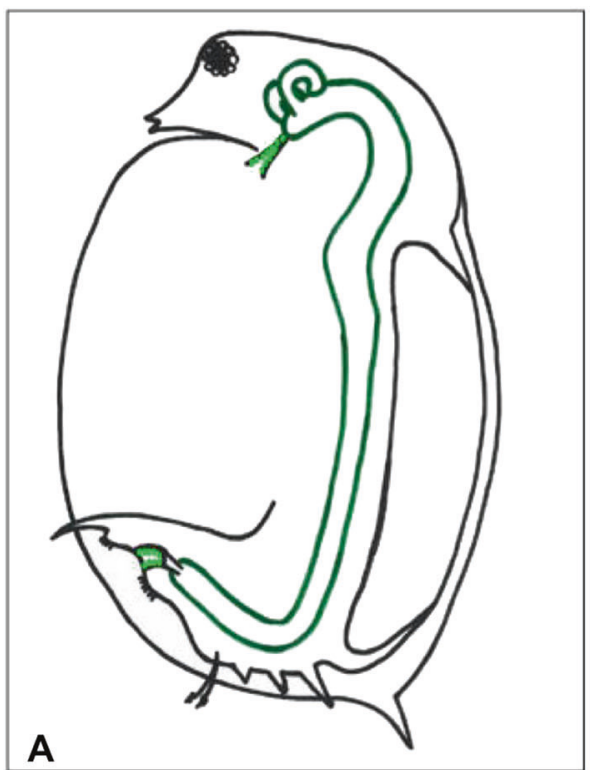

B

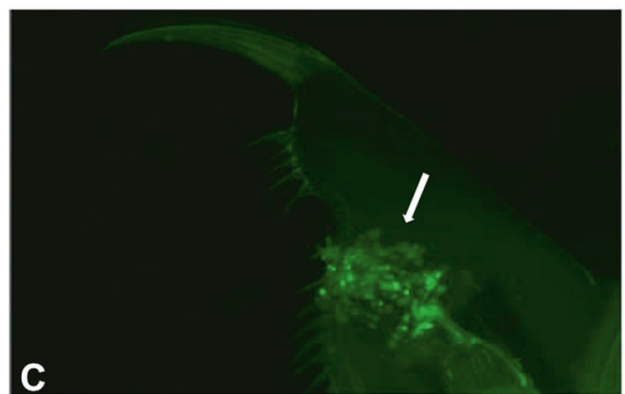

While we rarely have the necessary information to understand the role each step plays in the infection process and in the coevolution of host and parasite, this information does exist for the Daphnia magna-Pasteuria ramosa host-parasite system, where recent studies have identified the attachment step as crucial (Duneau et al. 2011, Luijckx et al. 2013; Metzger et al. 2016; Bento et al. 2017, reviewed in Ebert et al. 2016) (Fig. 1). Early in the infection process, $P$. ramosa spores are activated, shedding their protective exosporium. Next, the activated spores attach to the host's foregut (Fig. 1a,b) and penetrate into its body cavity where they reproduce and eventually kill the host. When foregut attachment fails, the host is resistant (Duneau et al. 2011). Variation in attachment success explains most variance in host-parasite interaction (Ebert et al. 2016). Studies have shown that the host-parasite interaction at the attachment step follows a matching-allele model (MAM), whereby infection of resistance can only occur when specific host and parasite alleles meet. MAM is one of the mechanisms that have been proposed to avoid the occurrence of supergenotypes, i.e., parasite genotype, which are universally infectious, or host genotypes, which are universally resistant (Lively and Dybdahl 2000; Hamilton 1980; Clarke 1976). Thus, in following a MAM, the Daphnia-Pasteuria host-parasite system fulfills a key assumption of coevolution by NFDS (Decaestecker et al. 2007; Duneau et al. 2011; Luijckx et al. 2012, Metzger et al. 2016; Bento et al. 2017).

The genetics of D. magna's resistance to Pasteuria spore attachment has received the most attention in the context of parasite genotypes $\mathrm{C} 1$ and $\mathrm{C} 19$ where one genomic region, the Pasteuria resistance (PR) locus, has been found to underlie variance in $D$. magna resistance to these $P$. ramosa genotypes. In addition, the natural variation at the PR locus has been found to match the predicted MAM (Bento et al. 2017). In this study, we report a newly isolated $P$. ramosa genotype, $\mathrm{P} 15$, that uses a different entry point into the host-the hindgut. We investigate D. magna's resistance to this Pasteuria isolate and discuss our findings on the infection mechanism and on the coevolutionary dynamics of this interaction.

\section{Methods}

\section{Hosts and parasites}

Two different $D$. magna genetic panels have been used in this study.

Diversity panel: The Daphnia magna Diversity Panel is a growing collection of $D$. magna genotypes collected across the entire species range (corresponding approximately to the entire Holarctic) with one genotype per population. All clones are kept clonally in laboratory standard medium (ADaM, [Klüttgen et al. 1994] modified by using only 5\% of the recommended selenium dioxide concentration) on a diet of green algae (Scenedesmus sp.) at a temperature of $20^{\circ} \mathrm{C}$ and a light: dark cycle of 16:8 (Luijckx et al. 2011). This panel has been used in previous studies (Roulin et al. 2013; Yampolsky et al. 2013; Seefeldt and Ebert 2019), but continues to expand with new D. magna clones. In this study, we used females from 174 clones. The diversity panel was used in this study to investigate the natural diversity of $D$. magna resistance to attachment and infection by $P$. ramosa $\mathrm{P} 15$ genotype. 
Recombinant panel: The F2-recombinant QTL panel used in this study was developed by Routtu et al. (2010) and was kept as clonal lines in the laboratory. In brief, this panel originated from the crossing of two divergent D. magna parent clones, one from a Finnish rock pool population (Xinb3) and the other from a pond near Munich, Germany (Iinb1). One F1 offspring was cloned and selfed to produce the F2-recombinant clones. These F2-recombinant clones were typed at about 1300 SNP markers to produce a genetic map (Routtu et al. 2014). Our F2-recombinant QTL panel in this study consisted of two subpanels: the core recombinant panel, a set of randomly chosen F2-recombinant clones, and the extended recombinant panel, a set of F2-recombinant clones selected for their susceptibility to the C19 Pasteuria ramosa genotype (among randomly chosen clones, only $25 \%$ are susceptible to this parasite genotype) (see Routtu et al. 2014 for more details). Here we used 208 core panel and 169 extended panel F2-recombinant clones to map and identify the genetic basis of $D$. magna resistance to $P$. ramosa $\mathrm{P} 15$.

Three $P$. ramosa parasite genotypes were used in this study: $\mathrm{C} 1$ and $\mathrm{C} 19$ clones were derived from natural isolates of P. ramosa from Moscow, Russia, and Gaarzerfeld, North Germany (see Luijckx et al. 2011), respectively, and have only been observed to attach to the foregut of the host. P15 was isolated from a sample collected in Heverlee, Belgium. It was not cloned, but was passaged multiple times in a susceptible D. magna host clone. For simplicity, we call it here the P15 genotype, but we cannot exclude the possibility that this isolate may contain some within isolate genetic variance. P15 typically attaches to the hindgut of the host, although we have observed a few cases where it attaches to the foregut.

\section{Attachment test}

The attachment test is a fluorescence-based assay that tests the ability of Pasteuria spores to attach to the fore- or hindgut of the host (Duneau et al. 2011) (Fig. 1b, c). Attachment indicates the parasite's ability to infect the host, while absence of attachment indicates resistant hosts (Duneau et al. 2011). Attachment is necessary for infection, but hosts may still be resistant if they can either prevent penetration (Duneau and Ebert 2012) or efficiently clear the infection (Hall et al. 2012) in later steps.

For the attachment tests, we exposed young host individuals to fluorescently labeled Pasteuria spores. After 30-60 min, the transparent hosts were observed under a fluorescent light to check if spores attached to their esophagus (foregut) or hindgut (Fig. 1b, c). More details of this method are provided in Duneau et al. (2011). Because the scoring of hindgut attachment phenotype is more difficult than the scoring of foregut attachment, there may also be an increased rate of false positives and negatives. We increased the number of replicates of the hindgut attachment (median $=12, \max =39$, and $\min =6$ ) assays relative to foregut attachment (median $=9, \max =27$, and $\min =6$ ) in order to increase our power to discern attachment success. All attachment tests were performed without the observer knowing the genotype of the animal being tested. All $D$. magna genotypes of the two genetic panels mentioned here were tested for their resistance to attachment by $P$. ramosa P15. For the purpose of these experiments described here, attachment was defined as a binary trait and clones classified as either resistant (no attachment) or susceptible (attachment).

\section{Infection test}

To test whether the infection assay protocol developed for foregut-attaching $P$. ramosa genotypes (Ben-Ami et al. 2010) would also result in successful P15 infections, we selected 40 clones from the QTL panel. Twenty one of these clones showed no P15 hindgut attachment in all tested replicates (P15_hindgut_R), and 19 clones showed P15 hindgut attachment in most or all replicates (P15_hindgut_S). The hindgut infection assay used here followed a modified version of previously published protocols for Pasteuria infections (Ben-Ami et al. 2010). The protocol modification consisted of individually cultured juvenile $D$. magna being exposed daily to a parasite spore dose high enough to infect susceptible hosts, i.e., those $D$. magna host genotypes prone to foregut-attaching Pasteuria with 90-100\% efficiency (Ben-Ami et al. 2010). This was done to avoid the removal of attached bacterial spore during molting (Duneau and Ebert 2012). In short, hosts were exposed to 50,000 parasite spores per day over 3 days, resulting in a total of 150,000 spores per animal. Exposed D. magna was fed daily with Scenedesmus sp. and kept individually in $80 \mathrm{~mL}$ medium (in $100 \mathrm{~mL}$ jars) under the same conditions as those in which it was raised (described above). After 40 days, we assessed the number of infected animals. Animals that died during the course of the experiment were not included in the analysis. The final dataset includes, on average, 19.03 replicates per clone. For the purpose of the experiments described in this paper, only the $D$. magna genotypes of the diversity panel were tested for their resistance to $P$. ramosa $\mathrm{P} 15$ infection.

\section{Genetic mapping}

Replicates (mean $=6$ replicates, range: $3-15$ ) of each of the 379 F2-recombinant clones of the F2 QTL panel were tested for P15 spore attachment (see "Attachment test" section). See Routtu et al. (2010) for a description of the structure and construction of the SNP array linkage map used. The 

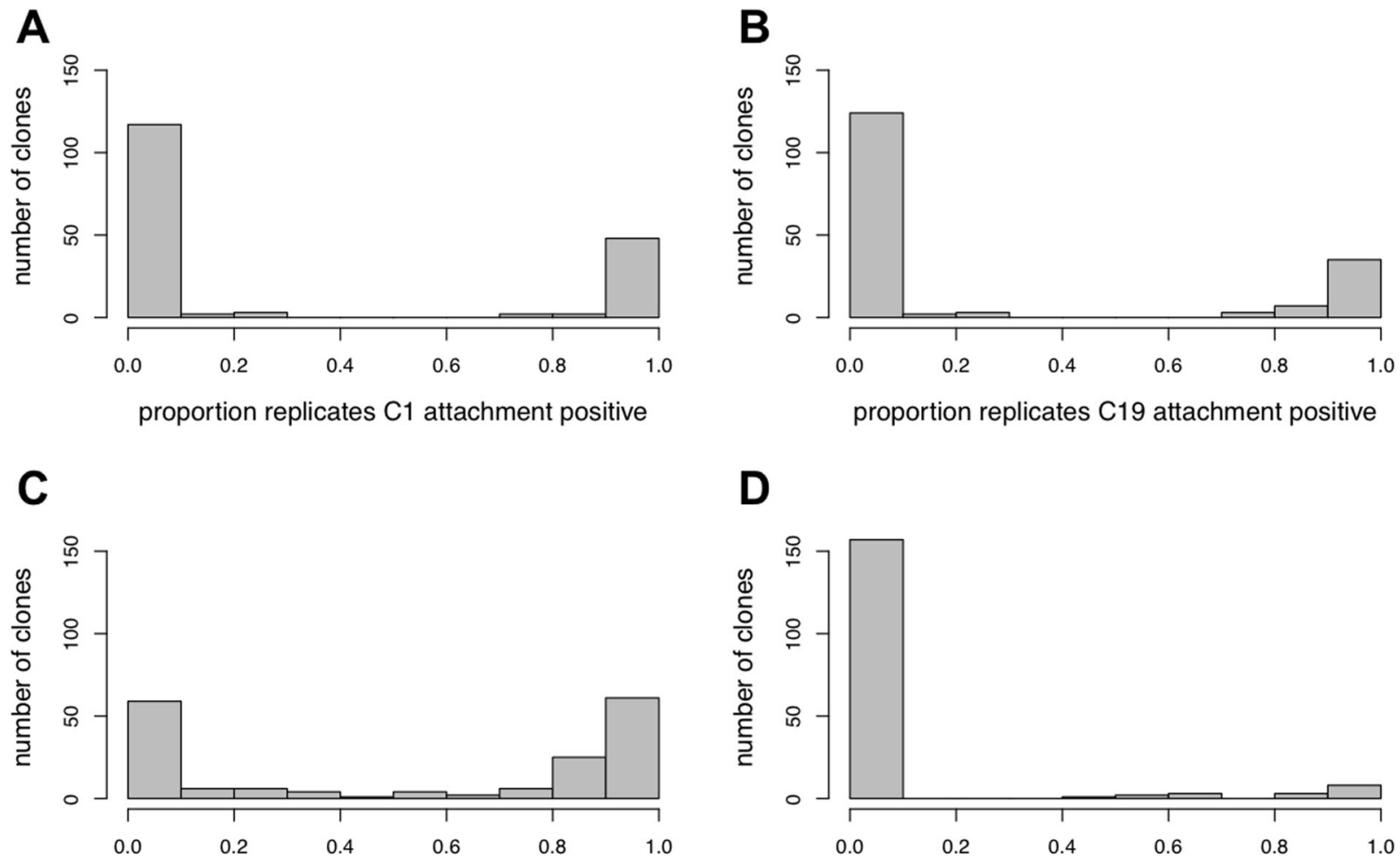

D

proportion replicates $\mathrm{P} 15$ hindgut attachment positive

Fig. 2 Variation of Pasteuria ramosa attachment success across 174 genotypes of the Daphnia magna diversity panel. Graphs show the proportion of positive attachment tests across replicates within genotype on the $\mathrm{x}$ axis and the number of $D$. magna genotypes (=clones)

QTL analysis was performed with $\mathrm{R}$ package $R /$ qtl version 1.29-2 (Broman et al. 2003), following the same analysis as described in Routtu and Ebert (2015) and Krebs et al. (2017) and using the same SNP map and QTL panel. In short, Haley-Knott regression (Knott, Haley 1992) was used for robustness and speed of analysis. The assumptions of the model were investigated and confirmed. To find epistatic interactions, we run scantwo (for multidimensional scans with a multiple-QTL model). Finally, using option fitqtl, a defined multiple-QTL model was analyzed (Broman et al. 2003). A genome-wide significance level was established using 10,000 permutation tests with significant $(\alpha=$ 0.05 ) LOD scores of 3.78. Analysis of variance was used to estimate the proportion of the total variance explained by the fitted models.

\section{Fine mapping}

To fine-map the D locus, clones generated in the Recombinant Panel were scored independently for attachment by $P$. ramosa P15 spores with two scoring methods: using a binary definition of attachment (resistant or susceptible) as described above and using a continuous definition of attachment where strength of attachment was classified by

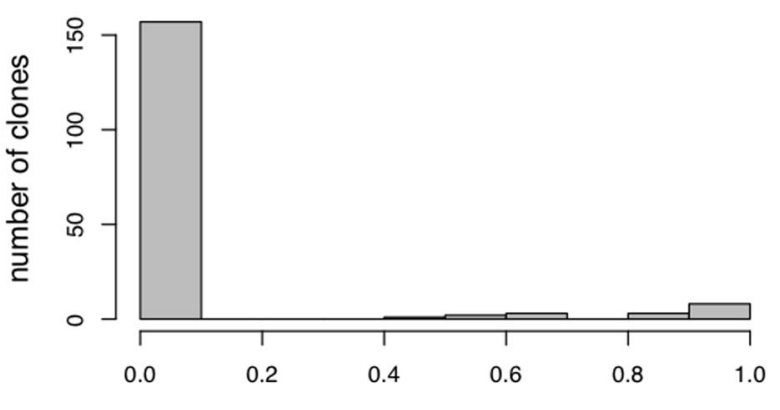

proportion replicates $\mathrm{P} 15$ foregut attachment positive

for each category on the $\mathrm{y}$ axis. The four graphs show $P$. ramosa $\mathrm{C} 1$ attachment to the host foregut (a), C19 to foregut (b), P15 to hindgut (c), and P15 to foregut (d).

the observer in a scale from 1 (no attachment) to 10 (strongest attachment). For further details see S1 Methods.

We validated the continuous definition of attachment by testing the correlation between the mean of the binary scoring and the median of the quantitative attachment scoring, using the 347 clones of the recombinant panel, which were tested with both methods. The results show that the binary and continuous methods are consistent with each other, and that the correlation is strong (Pearson $r=0.73, n$ $=347, p<0.001$ ).

For the fine mapping, we selected only those F2 clones that were scored either fully resistant or fully susceptible with both scoring methods before proceeding with the breakpoint mapping. For further details see S1 Methods.

\section{Sequencing, assembly, and annotation of D locus}

The methods for sequencing, assembling, and annotating the Xinb3 and Iinb1 D-locus haplotypes were identical to and are described in detail in Bento et al. (2017). In short, because the region around the QTL where D locus is located was poorly assembled in version 2.4 of the D. magna draft genome (http://wfleabase.org/), we undertook a number of additional sequencing and assembly methods in order to better resolve 
the focal region. For Xinb3, we generated high- coverage ( $60 \times)$ PacBio sequencing in order to perform de novo genome assembly. For linb1, we took a hybrid Illumina shortread/PacBio long-read approach, generating $\sim 80 \times 125 \mathrm{bp} \mathrm{PE}$ Illumina coverage and $\sim 15 \times$ PacBio long-read coverage. We used the D. magna Xinb3 and linb1 haplotype sequences obtained to blast search homologies within and between haplotypes and other genomic regions.

In order to understand how expression of individual genes localized to the focal genome regions and to other parts of the genome differed between the Xinb3 and linb1 clones, we conducted a de novo transcriptome assembly of the dataset described in Orsini et al. (2016). Finally, we constructed a de novo annotation of each of the transcripts mapping to the $\mathrm{D}$ locus by performing blastx (nucleotide-to-protein) searches in the NCBI database. For details on the methods for DNA extraction, genomic sequencing and assembly, and de novo transcriptome assembly mapping annotation see S2 Methods.

\section{Results}

\section{$P$. ramosa P15 attaches to $D$. magna hindgut and causes infection}

Of the 174 genotypes from the diversity panel that we tested for $P$. ramosa $\mathrm{P} 15$ attachment, most cases of positive spore attachment occurred in the hindgut of the host (Fig. 1c). Out of 2241 D. magna host individuals tested, 1197 (53\%) showed P15 attachment in the hindgut, while $163(7 \%)$ animals showed attachment to the host's foregut (Table S1). In contrast, testing the same $174 \mathrm{D}$. magna genotypes for attachment of $P$. ramosa clones $\mathrm{C} 1$ and $\mathrm{C} 19$, we observed only polymorphism for foregut attachment (Table S1), with no cases of hindgut attachment.

A hallmark of Pasteuria foregut attachment is that host genotypes (clones) either allow attachment or not, with very little within-clone phenotypic variance, i.e., inconsistent attachment test results between replicates from the same host genotype are rare (Duneau et al. 2011). However, P15 attachment to the hindgut appeared more variable: out of 174 D. magna genotypes tested, 59 (34\%) were attachmentnegative in all replicates, 47 (27\%) were attachment-positive in all replicates, and 68 genotypes (39\%) show variance among replicates of the same host genotype (with a minimum of six and an average of 12.8 individuals tested per host genotype) (Fig. 2). In testing the same 174 clones for $\mathrm{C} 1$ and $\mathrm{C} 19$ attachment, we found no within-clone variance for $\mathrm{C} 1$ and little variance for $\mathrm{C} 19$ attachment $(<10 \%$ of host clones, Fig. 2). Thus, we observed more quantitative variance for $P$. ramosa P15 hindgut attachment than for $\mathrm{C} 1$ and $\mathrm{C} 19$ foregut attachment. Nevertheless, the distribution of P15 attachment frequencies showed a pronounced bimodality (Fig. 2).
P. ramosa $\mathrm{P} 15$ attachment to the foregut was observed in about $7 \%$ of the Diversity panel host individuals. P15 foregut attachment was also variable within clones, but clearly bimodal (Fig. 2). Of those 17 D. magna genotypes where foregut attachment occurred in at least some replicates, eight showed attachment in all replicates (Fig. 2). Twelve of the 17 were resistant to P15 hindgut attachment, while the remaining five were able to attach to both the foregut and the hindgut (Table S1).

Attachment of $P$. ramosa $\mathrm{C} 1$ and $\mathrm{C} 19$ genotype spores to D. magna foregut was shown to be necessary for successful infections; indeed, infection nearly always follows $\mathrm{C} 1$ and C19 spore attachment (Duneau et al. 2011). We tested whether this held true for P. ramosa P15 attachment to the D. magna hindgut (Table S2) by using P15 attachmentpositive and P15 attachment-negative clones from a standing QTL panel (Routtu et al. 2014) and exposing 40 host clones to infectious P15 spores. The P15 attachment-negative clones remained largely uninfected (median infection rate: 0 , range: 0-22.2\%), whereas most P15 attachment-positive clones showed infection (median infection rate: $42.1 \%$, range: $0-94.4 \%$ ) (Fig. 3). We found a strong correlation between the attachment results and successful infection (Spearman's rho $=0.729, P<0.001, n=40$ ).

\section{QTL analysis and Mendelian segregation}

Our investigation into the genetic architecture of D. magna resistance to P15 hindgut attachment using the F2-

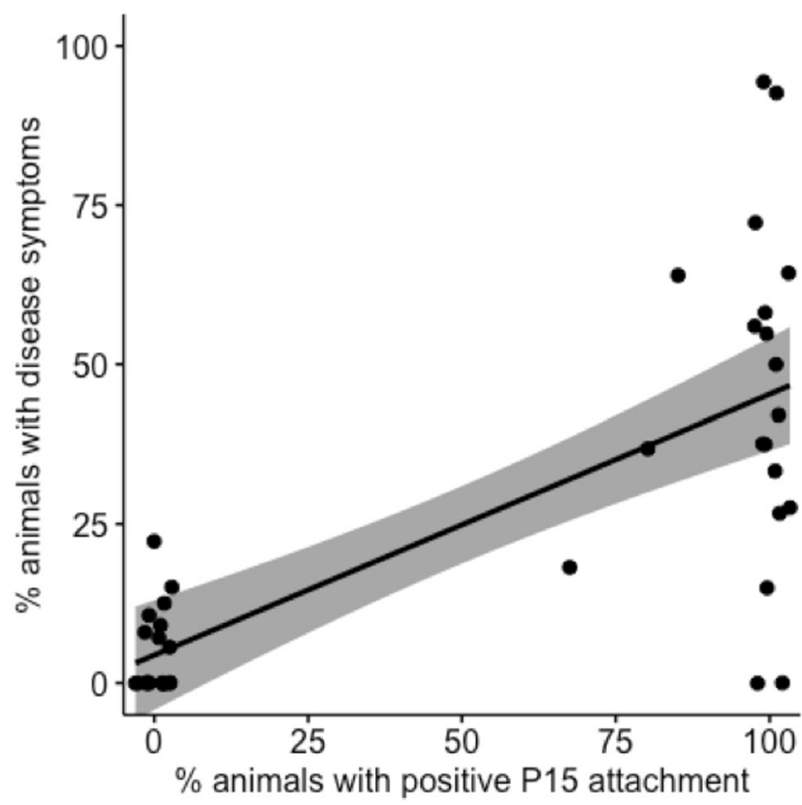

Fig. 3 Correlation of Pasteuria ramosa P15 attachment to the hindgut and the percentage of exposed animals that became infected. P15 hindgut attachment and infection success in 40 Daphnia magna genotypes (Spearman's rho $=0.73, p<0.001, n=40$ ). 
A

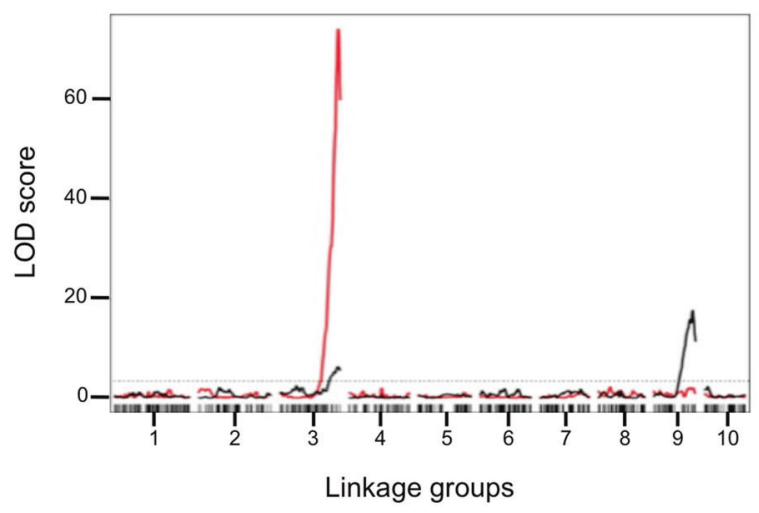

Fig. 4 Quantitative Trait Locus analysis of P. ramosa attachment to the host Daphnia magna. a LOD scores for Pasteruia attachment plotted against the entire genome of Daphnia magna. P. ramosa C19 attachment to the Daphnia magna foregut shown in red and $P$. ramosa P15 attachment to the hindgut shown in black. The dashed line represents the significance threshold at $P=0.05$. b Effect plot for P15

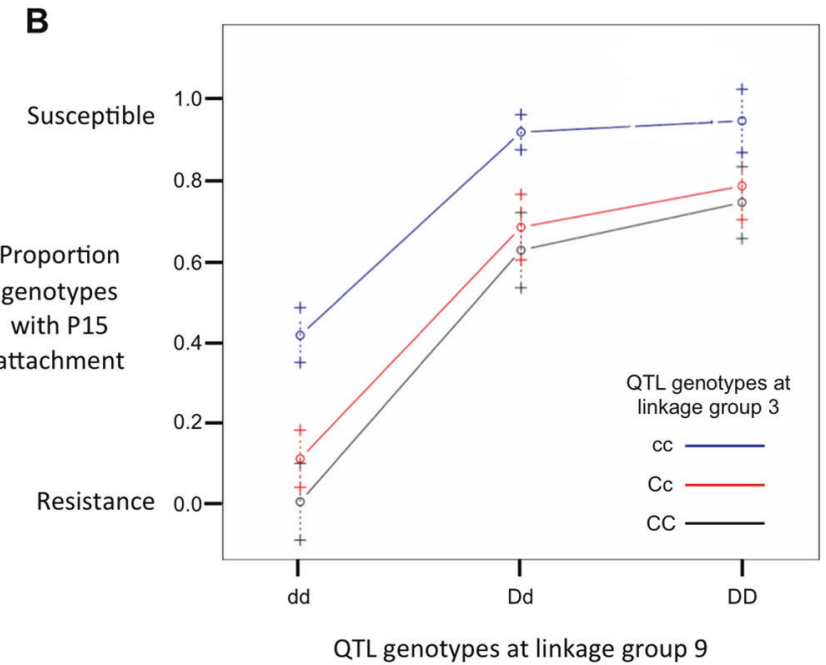

attachment of host genotypes at QTL detected in $\lg 3$ and $\lg 9$. The German parent clone (Iinb3) of the QTL panel has genotype CC at $\lg 3$ and dd at $\lg 9$, whereas the Finnish parent clone (Xinb3) has genotype $\mathrm{cc}$ at $\lg 3$ and DD at $\lg 9$. Variation shown along the $\mathrm{x}$ axis corresponds to the $\mathrm{D}$ locus, while the three colored lines represent variation at the C locus.
Table 1 Quantitative Trait Loci as detected by analysis of Pasteuria ramosa $\mathrm{P} 15$ attachment to Daphnia magna.

\begin{tabular}{llrrrrrl}
\hline & df & SS & LOD & \%var & $F$ value & $P$ value $\left(\mathrm{Chi}^{2}\right)$ & $P$ value $(\mathrm{F})$ \\
\hline Lg 3 SNP: scaffold00288_965 & 6 & 374.55 & 14.34 & 12.29 & 11.84 & $<0.001$ & $5.05 \mathrm{e}^{-12}$ \\
Lg 9 SNP: scaffold02269_730 & 6 & 957.68 & 32.21 & 31.42 & 30.28 & $<0.001$ & $<2 \mathrm{e}^{-16}$ \\
Lg 3 $\times \lg 9$ & 4 & 16.07 & 0.6771 & 0.53 & 0.76 & 0.54 & 0.551 \\
\hline
\end{tabular}

recombinant panel suggested that P15 attachment is dominant, as the F1 clone showed attachment. The parent clone Xinb3 has perfect attachment to P15, whereas parent clone Iinb1 has no attachment. Of the 377 F2 clones of the QTL panel, $51 \%$ of the genotypes showed within-clone variance, whereas 37\% showed attachment in all replicates, and 12\% showed no P15 spore attachment (Table S3).

The QTL analysis revealed two large-effect loci associated with P15 attachment in D. magna that together accounted for $42.1 \%$ of the total observed variance within the mapping population (Fig. 4a) (Table 1). One locus explained $11.8 \%$ of the variance and co-localizes with the previously described foregut attachment QTL on linkage group (lg) 3 (Routtu and Ebert 2015). This $\lg$ has been shown to harbor the PR locus for genetic variance in resistance to foregut attachment by $\mathrm{C} 1$ and $\mathrm{C} 19$ (Bento et al. 2017). The second QTL was found on $\lg 9$ and explained $30.3 \%$ of the total variance within the mapping population (Table 1). The effect plot (Fig. 4b) shows that the allele from the susceptible Finnish parent clone (Xinb3) at the second QTL is dominant for positive attachment and the allele from the resistant German parent clone (linb1) is recessive for no attachment. In maintaining our previous nomenclature of $P$. ramosa resistance loci (Metzger et al.
2016; Bento et al. 2017), we named this resistance locus the $\mathrm{D}$ locus. The interaction between the two QTLs on $\lg 3$ and $\lg 9$ was not significant (Table 1). Thus, there is no evidence for epistasis, and the joint effect of the two QTLs is therefore considered to be additive (Fig. 4b).

As seen in the natural host isolates (Fig. 2), there was within-clone phenotypic variance among the F2 clones for P15 attachment (Table S3). However, overall attachment frequencies for P15 and C19 showed clear bimodal distributions. By categorizing attachment-positive clones (P15_hindgut_S) as those in which more than 50\% of the replicates showed attachment, and attachment-negative clones (P15_hindgut_R) as all other clones, we were able to test the F2 clone data for Mendelian segregation. The F2 core panel $(n=208)$ showed Mendelian segregation for resistance to $\mathrm{C} 19$, with $73.6 \%$ of the $\mathrm{F} 2$ clones being $\mathrm{C} 19$ negative and $26.4 \%$ being C19-positive (Table 2), consistent with a 3:1 Mendelian segregation ratio $\left(\chi^{2}=0.865\right.$, $P=0.715)$. C19 attachment-positive clones have the CC or Cc genotype at the $\mathrm{C}$ locus and C19 attachment-negative clones are homozygotes for the recessive c allele (Metzger et al. 2016; Bento et al. 2017).

The 169 F2 clones of the extended QTL panel, which includes only C19 attachment-positive clones (i.e., 
Table 2 D. magna F2recombinant panel results for the core and the extended panel and for the genetic model for $P$. ramosa $\mathrm{C} 19$ and $\mathrm{P} 15$ genotypes.

Table 3 Single-nucleotide polymorphism marker sequences.

\begin{tabular}{|c|c|c|}
\hline \multicolumn{3}{|l|}{ Core panel (208 F2 clones) } \\
\hline & P15 attachment negative & P15 attachment positive \\
\hline & D-locus genotype: dd & D-locus genotype: DD or Dd \\
\hline $\begin{array}{l}\mathrm{C} 19 \text { attachment negative }-\mathrm{C} \text { - } \\
\text { locus genotype: } \mathrm{CC} \text { or } \mathrm{Cc}\end{array}$ & $\begin{array}{l}\text { C19_P15 resistotype: RR } \\
\text { genotype: CCdd or Ccdd } 31.25 \% \\
(n=65)\end{array}$ & $\begin{array}{l}\text { C19_P15 resistotype: RS genotype: } \\
\text { CCDD, CcDD, CCDd, or CcDd } \\
42.3 \%(n=88)\end{array}$ \\
\hline $\begin{array}{l}\text { C19 attachment positive C- } \\
\text { locus genotype: cc }\end{array}$ & $\begin{array}{l}\text { C19_P15 resistotype: SR } \\
\text { genotype: ccdd } 4.81 \%(n=10)\end{array}$ & $\begin{array}{l}\text { C19_P15 resistotype: SS genotype: } \\
\text { ccDD or } \operatorname{ccDd} 21.6 \%(n=45)\end{array}$ \\
\hline \multicolumn{3}{|l|}{ Extended panel (169 F2 clones) } \\
\hline & P15 attachment negative & P15 attachment positive \\
\hline & D-locus genotype: dd & D-locus genotype: DD or Dd \\
\hline $\begin{array}{l}\text { C19 attachment positive C- } \\
\text { locus genotype: cc }\end{array}$ & $\begin{array}{l}\text { C19_P15 resistotype }=\mathrm{SR} \\
\text { genotype: ccdd } 22.5 \%(n=38)\end{array}$ & $\begin{array}{l}\text { C19_P15 resistotype }=\mathrm{SS} \text { genotype: } \\
\text { ccDD or ccDd } 77.5 \%(n=131)\end{array}$ \\
\hline
\end{tabular}

The assessed resistotype (C19 P15), the inferred genotypes for the C locus (alleles: C and c) and the D locus (alleles D and d), and the percentage of host F2 clones for each resistotype are given. Capital letters of the inferred genotypes indicate dominant alleles. The data underlying this table are presented in Table S3.

\begin{tabular}{ll}
\hline Marker ID, comment & Sequence \\
\hline scaffold00288_965, best hit on $\lg 3$ & TTGTTAAAGTCCATTGTaAGTGTTTAAGTAGCAAA \\
scaffold02269_730, best hit on $\lg 9$, and & CATTTCGTCCTGAAaATATGTCACATTGTGTT
\end{tabular}

flanking SNP on lg9

scaffold01547_75, flanking SNP on $\lg 9$ AaTAAAATCTTAAAAACACaAAAGAAAATTCGTCCAA

Lowercase letters indicate SNPs. Flanking SNPs mark the markers closed to the D locus on $\lg 9$. homozygote cc), showed Mendelian segregation for P15 attachment resistance, with $77.5 \%$ being P15-positive and $22.5 \%$ being P15-negative (Table 2, extended panel). This coincides with a 3:1 Mendelian segregation ratio, with positive attachment (susceptibility) being dominant $\left(\chi^{2}=\right.$ $0.226, P=0.682$ ). Thus, we can infer that $\mathrm{P} 15$ attachmentpositive clones have DD or Dd genotypes at the newly defined D locus, and P15 attachment-negative clones have the recessive dd genotype at the D locus. However, in the core QTL panel, the proportion of P15 attachment-positive clones is only $63.9 \%$ (Table 2), representing a significant departure from the Mendelian segregation ratio $\left(\chi^{2}=5.086\right.$, $P<0.001$ ). If we consider only $\mathrm{F} 2$ clones that are $\mathrm{C} 19$ attachment-negative (genotypes $\mathrm{CC}$ or $\mathrm{Cc}$ ), this number declines further to $42.5 \%$ of clones being P15 attachmentpositive (Table 2). Thus, the dominant resistance allele at the $\mathrm{C}$ locus in $\lg 3$ increases the likelihood that the host genotype will show resistance to P15 attachment, a result consistent with the effect plot of the QTL analysis (Fig. 4b).

\section{Fine mapping and genomic characterization of the D locus}

Following the scoring of the attachment of $P$. ramosa P15 spores to the hindgut of the D. magna F2-recombinant clones with the binary and the qualitative method (Table S4), we selected only those D. magna clones that were fully susceptible or fully resistant with both scoring methods and were left with 112 genotypes (Table S5). The breakpoint mapping using these genotypes determined the boundaries of the large-effect QTL on $\lg 9$ where the D locus is located. The genomic region, which was identified, has $\sim 13 \mathrm{~kb}$ between SNP markers scaffold01547_75 and scaffold02269_730 (Table 3) (Table S5). We used PacBio long reads to sequence and assemble de novo the D-locus haplotype in the parental clones (Xinb3 and linb1) of the F2 QTL panel, designating these two haplotypes as $\mathrm{xD}$ locus (Xinb3 parent clone) and iD locus (Iinb1 parent clone), respectively.

By producing a de novo D. magna transcriptome and using reciprocal blasts between the D. magna transcriptome and the newly assembled D-locus haplotypes to map and annotate expressed genes in this region, we found six expressed genes in the xD-locus haplotype (Table 4).

In comparing the RNAseq database produced by Orsini et al. (2016) (based on the whole-body gene expression of the Xinb3 and Iinb1 parent clones of our QTL panel) with the gene expression at the $\mathrm{D}$ locus, we found that one transcript significantly upregulated in the $\mathrm{xD}$ locus, and one transcript that was significantly downregulated. The two transcripts, which were differentially regulated, correspond to two isoforms of a $D$. magna-probable ATP-dependent RNA helicase spindle E. Transcript TRINITY_DN20188_c3_g1_i1 (4337 bp long), which is upregulated in the Xinb3 P15-susceptible host clone, and the transcript TRINITY_DN20188_c3_g1_i2 (1408 bp long), 
Table 4 Daphnia magna expressed transcripts and predicted genes mapping to the D locus.

\begin{tabular}{llll}
\hline Predicted gene & Transcript & Length (bp) & $\begin{array}{l}\text { Up/downregulated in } \\
\text { Xinb3 parental clone }\end{array}$ \\
\hline Dm UP LOC116927272 ncRNA & TRINITY_DN1103_c0_g1_i1 & 871 & NS \\
$\begin{array}{l}\text { Dm-probable ATP-dependent } \\
\text { RNA helicase spindle E }\end{array}$ & TRINITY_DN20188_c3_g1_i1 & 4337 & Upregulated \\
& TRINITY_DN20188_c3_g1_i2 & 1408 & Downregulated \\
Dm calcyclic binding protein- & TRINITY_DN358_c0_g1_i1 & 340 & NS \\
like mRNA & TRINITY_DN20044_c2_g1_i6 & 2368 & NS \\
$\begin{array}{l}\text { Daphnia magna protein SMG7- } \\
\text { like }\end{array}$ & TRINITY_DN10965_c0_g1_i1 & 1392 & NS \\
$\begin{array}{l}\text { Dm UP LOC116927249 ncRNA } \\
\text { Dm MIT domain protein 1- }\end{array}$ & TRINITY_DN6503_c0_g1_i1 & 628 & NS \\
like mRNA & TRINITY_DN10965_c0_g1_i1 & 1392 & NS \\
\hline NS nonsignificant. & & & \\
\hline
\end{tabular}

$N S$ nonsignificant. which is downregulated in the Xinb3 P15-susceptible clone. Neither of them has, to our knowledge, been associated with resistance against a pathogen.

\section{Discussion}

Bacterial attachment to the host's foregut has been shown to be a crucial step in the infection process of $D$. magna by $P$. ramosa parasites. Failure in this step blocks infection altogether, whereas success nearly guarantees infection (Duneau et al. 2011). Here we describe an alternate location of parasite entry into the D. magna host-attachment to the host's hindgut. This route has not been described before and is used by a different genotype $(\mathrm{P} 15)$ of $P$. ramosa. Interestingly, P15 is also able to use the foregut as an attachment site in some cases.

The discovery of a second entry point for $P$. ramosa into its host adds complexity to our understanding of the mechanisms and evolution underlying host resistance to Pasteuria infections. Our previous picture relied on the assumption of a linear stepwise infection process: (1) host-parasite encounter, (2) activation of the parasite endospore, (3) attachment to the host cuticle, (4) penetration of the host, (5) early and (6) late within-host growth phase, and (7) host death (Ebert et al. 2016, Hall et al. 2017). Our new finding suggests that this process bifurcates after the activation step (step 2), with parasites being able to attach (step 3) and penetrate (step 4) at one of two entry points, or, in some cases, at both points. Following host penetration, parasites that enter through both entry points start proliferating within the host's body cavity (steps 5 and 6), leading finally to the death of the host (step 7). Alternate host entry points are known from other host-parasite systems. For example, anthrax infections in humans are caused by Bacillus anthracis, which has multiple ways to enter a host, including lung entry after the spores are inhaled, cutaneous anthrax after skin contact with spores, and gastrointestinal anthrax after food-borne spore uptake (www. cdc.gov/anthrax/basics/how-people-are-infected.html). In the Daphnia system, the microsporidium Hamiltosporidium tvaerminnensis is able to enter the host by transovarial infections and through the ingestion of free spores with the food (Vizoso and Ebert 2005). Unless different infections routed are traded off with each other (Lipsitch et al. 1995), parasites generally benefit from having multiple ways to infect a host, as it increases their chances of infection and may reduce the chances for the host to evolve resistance. With the second entry route for Pasteuria that we describe here, neither a trade-off nor a widening of opportunities with different ecological conditions for the two routes of entry are known.

Both the foregut and the hindgut of D. magna are ectodermic tissues that are part of the animals' cuticle and that are molted in regular intervals throughout the host's life. The remaining section of the gut (the midgut) is of endodermic origin and is not molted. As the hind- and foregut tissue stems from a shared ectodermic origin (Schultz and Kennedy 1976) and possibly a shared genetic basis, the attachment mechanism might be conserved in the two infection routes. The observation that $\mathrm{P} 15$ can attach to both the hindgut and the foregut, and in some host genotypes even to both sites, further suggests a similarity of the two attachment sites. However, nothing is known about the cuticle surface of those sites in Daphnia. The general course of an infection is also similar at both sites. The typical disease symptoms - castration, gigantism, and a life span of 40-50 days post infection-that are observed in response to infections by foregut-attaching Pasteuria genotypes (Clerc et al. 2015; Ben-Ami 2017), are also observed in response to infection by the P15 isolate.

Our study also revealed an important difference between fore- and hindgut attachment: we observed that positive attachment (susceptibility) is recessive when tested with 
Pasteuria C19, but is dominant when tested with Pasteuria $\mathrm{P} 15$. This is, however, not entirely new for this system, as an earlier study demonstrated that in the A locus of the PR supergene (Bento et al. 2017) (which includes the A, B, and C loci), either resistance or susceptibility can show as dominant depending on the Pasteuria isolate used and the genotype at other loci (Luijckx et al. 2013). A further difference observed in P15 hindgut attachment is that attachment success followed a more quantitative, but still strongly bimodal, pattern (Fig. 2). About $40 \%$ of host clones isolated from natural populations showed within-clone phenotypic variance in hindgut attachment success for Pasteuria P15. For the foregut-attaching Pasteuria C1 and C19, this percentage is below $10 \%$ (Fig. 2). We also observed that the infection rate among P15 attachment-positive host clones was comparatively low and highly variable (Fig. 3). A few host clones remained uninfected even after being exposed to spore doses that would lead typically to nearly $100 \%$ infection success for the two foregutattaching parasites C1 and C19 (Duneau et al. 2011). Whether the reduced infection rate of P15 attachment-positive clones is caused by less-efficient attachment and host penetration, or by subsequent clearing of infections by the host's immune system (within-host defense), is not clear. Surprisingly, some clone replicates that were scored as consistently P15-hindgut attachment negative developed a full-blown infection after exposure to the spores. This finding may hint at a third, yet-tobe-discovered, entry point into the host.

The lower consistency in hindgut attachment may stem from differences in the attachment mechanism, with potentially weaker cell-to-cell attachment in the host hindgut. It may also be a consequence of the increased difficulties that infective spores have in reaching the hindgut attachment site. The passage through the host's entire intestinal tract represents an extra step in the infection process and may add variability in the interaction between parasite and host that reduces the number and quality of parasite spores reaching the attachment site. Indeed, as the outer shell (the protective exosporium) of the spores is removed before it is ingested by the host, the host's defense and the digestive system may alter the integrity of the activated, and hence unprotected, bacteria. Given these variables, stochastic effects likely play a larger role for Pasteuria attachment in the hindgut, although other factors may also contribute to attachment success, including the role of undetected minor-effect QTL.

\section{Hindgut attachment is genetically determined by two loci}

Previous genetic studies of natural variation in resistance to C1 and C19 attachment suggest that D. magna's genetic variance in resistance to these $P$. ramosa clones lies within a model of three linked loci (A, B, and C). These loci are localized in the PR locus, which was mapped on linkage group 3 ( $\lg 3$ ) (Routtu and Ebert 2015; Metzger et al. 2016; Bento et al. 2017). For one of these loci, the A locus, a matching allele matrix has been described (Luijckx et al. 2012; Bento et al. 2017). For the P15 clone, the QTL analysis of hindgut attachment revealed one locus that colocalizes within or close to the previously described largeeffect QTL on $\lg 3$ (Routtu and Ebert 2015), as well as a second locus, the D locus, that was newly discovered in this study. This D locus explains most genetic variance within our mapping population in P15 hindgut attachment. Our analysis suggests that the effect of these two QTLs is additive, although this conclusion might change with a larger sample size, as additivity is concluded from the absence of significant epistatic effects. Strong epistasis has been reported for the interaction between the A-, B-, and C locus (Metzger et al. 2016, Bento et al. 2017).

This PR-locus supergene, responsible for variation in foregut attachment on $\lg 3$, shows dramatic structural polymorphism between resistant and susceptible D. magna clones, including long sequences that are nonhomologous and apparently nonrecombining (Bento et al. 2017). Defined as a genomic region that hosts a number of genes involved in one phenotype, this supergene serves as a hotspot for adaptive evolution and, due to suppressed recombination, behaves genetically like a single gene (Schwander et al. 2014; Bento et al. 2017). The two PR-loci haplotypes differ by $\sim 55 \mathrm{~kb}$ and the nonhomologous regions stretch across more than $70 \mathrm{~kb}$ (34\% of the xPR locus and $46 \%$ of the iPR locus) (Bento et al. 2017).

In contrast, the D locus described here showed no major structural differences, and the two D-loci haplotypes are of similar length: xD locus is $13,059 \mathrm{bp}$ long, and iD locus is $12,751 \mathrm{bp}$ long. Also, in contrast to the PR locus, we could not find any extensive repeat structure in the D locus (Bento et al. 2017).

Taken together, this evidence indicates that the D locus is a very different type of genomic region from the unusual structure and polymorphism observed in PR locus, suggesting a different evolutionary and genetic history for these two Pasteuria resistance loci.

\section{The evolution of host-parasite interactions}

Our survey of a Holarctic sample of 174 D. magna genotypes indicates that resistance polymorphism for P15 attachment is widespread (found within Europe, Asia, North Africa, and North America) and thus should be considered in the evolution of Daphnia-Pasteuria interactions. Having two entry points reduces the strength of selection for resistance at each of the points, as the direct link between entry point and infection is weakened, especially when population infection rates are 
high and multiple infections are common. A further complication is that one of the QTLs described here explains genetic variance for both $\mathrm{C} 19$ foregut resistance and P15 hindgut resistance. Thus, the evolution of resistance at the two entry points is not independent from each other.

Our findings also have implications for the evolution of the parasite. Since attachment to the hindgut appears to be weaker and more subject to stochastic variation (as compared with foregut attachment), foregut-attaching parasites have an advantage over hindgut-attaching parasites. Within-host competition may further amplify this advantage, because it is likely to give the competitive edge to the (on average) earlier-infecting parasite genotype (the one with the higher force of infection). On the other hand, the new Pasteuria isolate, P15, does have an advantage in that it can attach to both fore- and hindgut, which increases its chances to encounter a susceptible host. It is expected that selection would favor this ability to use additional entry points, unless it comes with costs. Interestingly, we observed that of the 174 host genotypes tested, more than $50 \%$ are attachment positive for P15, while only about $30 \%$ were positive to attachment with $\mathrm{C} 1$ and $\mathrm{C} 19$ (Fig. 2). Thus, P15 may be able to compensate for its lower infectivity with a higher attachment rate.

As for coevolution in the Daphnia-Pasteuria model, this is one of the few examples where a MAM has been observed in a host-parasite system (Bento et al. 2017). This MAM is observed for the segregation of the A locus and only in a specific genetic background: the B locus must be fixed for the dominant $\mathrm{B}$ allele and the $\mathrm{C}$ locus for the recessive $\mathrm{c}$ allele (Bento et al. 2017; Luijckx et al. 2013). The A locus does not segregate in the F2recombinant QTL panel, and therefore we do not know if the D locus influences the MAM for the A locus. However, the interaction of the D locus with the C locus, which in turn is linked to the A locus, suggests that this might be possible. The increasing number of resistance loci being uncovered in this study system would lower the power of natural selection to change allele frequencies, while the importance of genetic recombination for the resistance phenotype increases. However, the ability to predict resistance phenotypes and the relative influence of selection and recombination in the natural history of the Daphnia-Pasteuria system is dependent on local diversity patterns. The diversity panel, which we used in this study, is made of genotypes collected from 174 populations from throughout the Holarctic, which maximizes genetic variance, but does not reflect diversity in a local population.

For the moment, we do not know how widespread parasites using different entry points or their combination are in natural populations. But we believe that the finding of P15 hindgut entry adds at least two important pieces to our understanding of the coevolutionary dynamics of the Pasteuria-Daphnia system. First, hosts and parasites are more diverse than previously known, therefore providing more opportunities for selection to act on. Second, with the finding of a resistance locus distant from the PR supergene, recombination becomes more important, as it creates further variability in host resistance. Even though this study adds further understanding of the interaction between D. magna and its parasite $P$. ramosa, the current picture still misses a lot of the natural variation present in hosts and parasites. Thus, it is not clear at the moment if the Red Queen may run faster or slower with the extra level of variation described here (Lively 2010).

\section{Data availability}

Raw PacBio reads originating from the xD- and iD-locus haplotypes can be accessed under NCBI BioProject \# PRJNA528268. Assembled haplotypes for the xD- and iDlocus can be found under GenBank accessions MK684166 and MK684167.

Acknowledgements We thank Jürgen Hottinger and Urs Stiefel for assistance in the laboratory. We thank Louis Du Pasquier for helpful discussions. Suzanne Zweizig improved the language of the paper. Camille Ameline produced the drawing in Fig. 1a. This work was supported by an EMBO long-term fellowship to GB and a Swiss National Science Foundation grant to DE. DD was supported by the French Laboratory of Excellence project "TULIP" (ANR-10-LABX41; ANR-11-IDEX0002-02).

Author contributions DD discovered the alternative route of infection. $\mathrm{DE}, \mathrm{DD}$, and GB conceived the study. GB conducted the experiments. GB, DE, and PDF analyzed the data. GB wrote the paper. All authors contributed to the writing.

\section{Compliance with ethical standards}

Conflict of interest The authors declare that they have no conflict of interest.

Publisher's note Springer Nature remains neutral with regard to jurisdictional claims in published maps and institutional affiliations.

Open Access This article is licensed under a Creative Commons Attribution 4.0 International License, which permits use, sharing, adaptation, distribution and reproduction in any medium or format, as long as you give appropriate credit to the original author(s) and the source, provide a link to the Creative Commons license, and indicate if changes were made. The images or other third party material in this article are included in the article's Creative Commons license, unless indicated otherwise in a credit line to the material. If material is not included in the article's Creative Commons license and your intended use is not permitted by statutory regulation or exceeds the permitted use, you will need to obtain permission directly from the copyright holder. To view a copy of this license, visit http://creativecommons. org/licenses/by/4.0/. 


\section{References}

Ben-Ami (2017) The virulence-transmission relationship in an obligate killer holds under diverse epidemiological and ecological conditions, but where is the tradeoff? Ecol Evol 7(24):11157-11166

Ben-Ami F, Ebert D, Regoes RR (2010) Pathogen dose infectivity curves as a method to analyze the distribution of host susceptibility: a quantitative effect of maternal effects after food stress and pathogen exposure. Am Nat 175(1):106-115

Bento G, Routtu J, Fields PD, Bourgeois Y, Du Pasquier L, Ebert D (2017) The genetic basis of resistance and matching-allele interactions in a host-parasite system: The Daphnia magna-Pasteuria ramosa model. PLoS Genet 13(2):e1006596

Broman KW, Wu H, Sen, Churchill GA (2003) R/qtl: QTL mapping in experimental crosses. Bioinformatics 19:889-890

Clarke B (1976) The ecological genetics of host-parasite relationships. In:Taylor AER, Muller R (eds) Genetic aspects of hostparasite relationships. Blackwell, London, p 87-103

Clerc M, Ebert D, Hall MD (2015) Expression of parasite genetic variation changes over the course of infection: implications of withinhost dynamics for the evolution of virulence. Proc Biol Sci 282 (1084):20142820

Decaestecker E, Gaba S, Raeymaekers JAM, Stoks R, Van Kerckhoven L, Ebert D, De Meester L (2007) Host-parasite "Red Queen" dynamics archived in pond sediment. Nature 450(7171):870-873

Duneau D, Ebert D (2012) The role of moulting in parasite defence. Proc Biol Sci 279(1740):3049-3054

Duneau D, Luijckx P, Ben-Ami F, Laforsch C, Ebert D (2011) Resolving the infection process reveals striking differences in the contribution of environment, genetics and phylogeny to host-parasite interactions. BMC Biol https://doi.org/10.1186/1741-7007-9-11

Ebert D (2008) Host-parasite coevolution: insights from the Daphniaparasite model system. Curr Opin Microbiol 11(3):290-301

Ebert D, Duneau D, Hall MD, Luijckx P, Andras JP, Du Pasquier L, Ben-Ami F (2016) A population biology perspective on the stepwise infection process of the bacterial pathogen Pasteuria ramosa in Daphnia. Adv Parasitol 91:265-310

Ejsmond MJ, Radwan J (2015) Red Queen processes drive positive selection on Major Histocompatibility Complex (MHC) genes. PLoS Comput Biol 11(11):e1004627

Hall MD, Bento G, Ebert D (2017) The evolutionary consequences of stepwise infection processes. Trends Ecol Evol 32(8):612-623

Hall MD, Ebert D (2012) Disentangling the influence of parasite genotype, host genotype and maternal environment on different stages of bacterial infection. In Proceedings of the Royal Society B: Biological Sciences 279(1741):3176-3183

Hamilton WD (1980) Sex versus non-sex versus parasite. Oikos 35:282-290

Klüttgen B, Dulmer U, Engels M, Ratte HT (1994) ADaM, an artificial freshwater for the culture of zooplankton. Water Res 28:743-746

Knott SA, Haley CS (1992) Maximum likelihood mapping of quantitative trait loci using full-sib families. Genetics 132(4):1211-1222

Krebs M, Routtu J, Ebert D (2017) QTL mapping of a natural genetic polymorphism for long-term parasite persistence in Daphnia populations. Parasitology 144(13):1686-1694

Lievens EJP, Perreau J, Agnew P, Michalakis Y, Lenormand T (2018) Decomposing parasite fitness reveals the basis of specialization in a two-host, two-parasite system. Evol Lett 2(4):390-405

Lipsitch M, Nowak MA, Ebert D, Ray RM (1995) The population dynamics of vertically and horizontally transmitted parasites. Proc Biol Sci 250(1359):321-327
Lively CM (2010) A review of Red Queen models for the persistence of obligate sexual reproduction. J Hered 101(Suppl 1): S13-S20

Lively CM, Dybdahl MF (2000) Parasite adaptation to locally common host genotypes. Nature 405(6787):679-681

Luijckx P, Fienberg H, Duneau D, Ebert D (2012) Resistance to a bacterial parasite in the crustacean Daphnia magna shows Mendelian segregation with dominance. Heredity 108(5):547-551

Luijckx P, Fienberg H, Duneau D, Ebert D (2013) A matching-allele model explains host resistance to parasites. Curr Biol 23 (12): $1085-1088$

Luijckx P, Ben-Ami F, Mouton L, Du Pasquier L, Ebert D (2011) Cloning of the unculturable parasite Pasteuria ramosa and its Daphnia host reveals extreme genotype-genotype interactions. Ecol Lett 14(2):125-131

Metzger CM, Luijckx P, Bento G, Mariadassou M, Ebert D (2016) The Red Queen lives: epistasis between linked resistance loci. Evolution 70(2):480-487

van Oosterhout C (2009) A new theory of MHC evolution: beyond selection on the immune genes. Proc Biol Sci 276(1657):657-665

Orsini L, Gilbert D, Podicheti R, Jansen M, Brown JB, Solari OS et al. (2016) Daphnia magna transcriptome by RNA-seq across 12 environmental stressors. Sci Data 3:160030

Papkou A, Gokhale CS, Traulsen A, Schulenberg H (2016) Hostparasite coevolution: why changing population size matters. Zool (Jena) 119(4):330-338

Roulin AC, Routtu J, Hall MD, Janicke T, Colson I, Haag CR, Ebert D (2013) Local adaptation of sex induction in a facultative sex crustacean: insights from QTL mapping and natural populations of Daphnia magna. Mol Ecol 22(13):3567-3579

Routtu J, Ebert D (2015) Genetic architecture of resistance in Daphnia hosts against two species of host-specific parasite. Heredity 114 (2):241-248

Routtu J, Jansen B, Colson I, De Meester L, Ebert D (2010) The firstgeneration Daphnia magna linkage map. BMC Genom 11:508

Routtu J, Hall MD, Albere B, Beisel C, Bergeron RD, Chaturvedi A et al. (2014) An SNP-based second-generation genetic map of Daphnia magna and its application to QTL analysis of phenotypic traits. BMC Genom 15:1033

Schmid-Hempel P (2011) Evolutionary Parasitology, 1st edn. Oxford University Press (Oxford, UK)

Schultz TW, Kennedy JR (1976) The fine structure of the digestive system of Daphnia pulex (Crustacea: Cladocera). Tissue Cell 8 (3):479-490

Schwander T, Libbrecht R, Keller K (2014) Supergenes and complex phenotypes. Current Biology 24(7):R288-R294

Seefeldt L, Ebert D (2019) Temperature- versus precipitationlimitation shape local temperature tolerance in a Holarctic freshwater crustacean. Proc Biol Sci 286(1907):20190929

Tiffin P, Moeller DA (2006) Molecular evolution of plant immune genes. Trends Genet 22(12):662-670

Vizoso DB, Lass S, Ebert D (2005) Different mechanisms of transmission of the microsporidium Octosporea bayeri: a cocktail of solution for the problem of parasite permanence. Parasitology 130 (Pt5):501-509

Wilfert L, Jiggins FM (2013) The dynamics of reciprocal selective sweeps of host resistance and parasite counter adaptation in. Drosoph Evolution 67(3):761-773

Yampolski LY, Schaer TM, Ebert D (2013) Adaptive phenotypic plasticity and local adaptation for temperature tolerance in freshwater zooplankton. Proc Biol Sci 281(1776):20132744 